\title{
Fekete-Szegö Coefficient Functional for Certain Subclasses of Close-to-Star Functions
}

\author{
B. S. Mehrok ${ }^{1}$ and Gagandeep Singh ${ }^{2}$ \\ ${ }^{1}$ Department of Mathematics, Khalsa College, Amritsar, Punjab 143001, India \\ ${ }^{2}$ Department of Mathematics, DIPS College (Co-Educational), Dhilwan, Kapurthala, Punjab 143401, India \\ Correspondence should be addressed to Gagandeep Singh; kamboj.gagandeep@yahoo.in
}

Received 8 April 2013; Accepted 10 June 2013

Academic Editor: JinLin Liu

Copyright (c) 2013 B. S. Mehrok and G. Singh. This is an open access article distributed under the Creative Commons Attribution License, which permits unrestricted use, distribution, and reproduction in any medium, provided the original work is properly cited.

We introduce some subclasses of close-to-star functions defined by subordination and obtain sharp upper bounds of the functional $\left|a_{3}-\mu a_{2}^{2}\right|, \mu$ real, for an analytic function $f(z)=z+\sum_{n=2}^{\infty} a_{n} z^{n},|z|<1$, belonging to these sub-classes.

\section{Introduction}

Let $\Omega$ be the class of bounded functions of the following form:

$$
w(z)=\sum_{n=1}^{\infty} d_{n} z^{n}
$$

which are analytic in the unit $\operatorname{disc} E=\{z:|z|<1\}$ and satisfy the conditions $w(0)=0$ and $|w(z)|<1$.

It is known (see [1]) that

$$
\left|d_{1}\right| \leq 1, \quad\left|d_{2}\right| \leq 1-\left|d_{1}\right|^{2} .
$$

Let $A$ denote the class of functions of the following form:

$$
f(z)=z+\sum_{n=2}^{\infty} a_{n} z^{n}
$$

which are analytic in $E$. Let $S$ be the class of functions of the form (3) which are analytic univalent in $E$.

We will concentrate on the coefficient problem for the subclasses of $A$. In 1916, Bieberbach [2] proved that $\left|a_{2}\right| \leq 2$ for $f(z) \in S$ as an elementary corollary area theorem. He conjectured that, for each function $f(z) \in S,\left|a_{n}\right| \leq n$; the equality holds for the Koebe function $k(z)=z /(1-z)^{2}$, which maps the unit disc $E$ onto the entire complex plane minus the slit along the negative real axis from $-1 / 4$ to $-\infty$. De Branges [3] solved the Bieberbach conjecture in 1985. The contribution of Löwner [4] in proving that $\left|a_{3}\right| \leq 3$ for the class $S$ was huge.

With the known estimates $\left|a_{2}\right| \leq 2$ and $\left|a_{3}\right| \leq 3$, it was natural to seek some relation between $a_{3}$ and $a_{2}^{2}$ for the class $S$. This thought prompted Fekete and Szegö [5] and they used Löwner's method to prove the following well-known result for the class $S$ : if $f(z) \in S$, then

$$
\left|a_{3}-\mu a_{2}^{2}\right| \leq \begin{cases}3-4 \mu & \text { if } \mu \leq 0 \\ 1+2 \exp \left(\frac{-2 \mu}{1-\mu}\right) & \text { if } 0 \leq \mu \leq 1 \\ 4 \mu-3 & \text { if } \mu \geq 1\end{cases}
$$

The inequality (4) plays a very important role in determining estimates of higher coefficients for some subclasses of $S$ (see Chichra [6], Babalola [7]).

Next, we define some subclasses of $A$ and obtain the analogy of (4).

We denote by $S^{*}$ the class of univalent star-like functions $g(z)=z+\sum_{n=2}^{\infty} b_{n} z^{n} \in A$ which satisfy the following condition:

$$
\Re\left(\frac{z g^{\prime}(z)}{g(z)}\right)>0, \quad z \in E .
$$


We denote by $K$ the class of convex univalent functions $h(z)=z+\sum_{n=2}^{\infty} c_{n} z^{n} \in A$ which satisfy the condition

$$
\Re\left(\frac{\left(z h^{\prime}(z)\right)^{\prime}}{h^{\prime}(z)}\right)>0, \quad z \in E .
$$

Let $f$ and $g$ be two analytic functions in $E$. Then, $f$ is said to be subordinate to $g$ (symbolically $f \prec g$ ) if there exists a bounded function $w(z) \in \Omega$, such that $f(z)=g(w(z))$.

A function $f(z) \in A$ is said to be close to star if there exists a function $g(z) \in S^{*}$ such that

$$
\mathfrak{R}\left(\frac{f(z)}{g(z)}\right)>0, \quad z \in E
$$

The class of close to star functions was introduced by Reade [8] and is denoted by $C^{*}$. A close to star function need not be univalent. The immediate shoot of $C^{*}$ is the following subclass:

$$
C_{1}^{*}=\left\{f(z) \in A: \mathfrak{R}\left(\frac{f(z)}{h(z)}\right)>0, h(z) \in K, z \in E\right\} .
$$

In this paper, we consider the following subclasses of close-to-star functions:

$$
\begin{aligned}
C^{*}(A, B)= & \left\{f(z) \in A: \frac{f(z)}{g(z)} \prec \frac{1+A z}{1+B z},\right. \\
& \left.\quad g(z) \in S^{*},-1 \leq B<A \leq 1, z \in E\right\} .
\end{aligned}
$$

The class $C^{*}(A, B)$ was introduced and studied by Mehrok et al. [9]. In particular, $C^{*}(1,-1) \equiv C^{*}$,

$$
\begin{aligned}
C_{1}^{*}(A, B)= & \left\{f(z) \in A: \frac{f(z)}{h(z)} \prec \frac{1+A z}{1+B z},\right. \\
& h(z) \in K,-1 \leq B<A \leq 1, z \in E\},
\end{aligned}
$$

is the class introduced and studied by Mehrok et al. [10]. Obviously, $C_{1}^{*}(1,-1) \equiv C_{1}^{*}$.

Hummel [11] proved the conjecture of $V$. Singh that $\mid c_{3}-$ $c_{2}^{2} \mid \leq 1 / 3$ for the class $K$. Keogh and Merkes [12] obtained the estimates (4) for the classes $S^{*}$ and $K$. We obtain sharp upper bounds of the functional $\left|a_{3}-\mu a_{2}^{2}\right|$ for functions belonging to the classes $C^{*}(A, B)$ and $C_{1}^{*}(A, B)$.

\section{Preliminary Lemmas}

Lemma 1. Let $g(z) \in S^{*}$. Then,

$$
\left|b_{3}-\mu b_{2}^{2}\right| \leq \begin{cases}3-4 \mu & \text { if } \mu \leq \frac{1}{2} \\ 1 & \text { if } \frac{1}{2} \leq \mu \leq 1 \\ 4 \mu-3 & \text { if } \mu \geq 1\end{cases}
$$

This lemma is due to Keogh and Merkes [12].

Lemma 2. Let $h(z) \in K$. Then,

$$
\left|c_{3}-\mu c_{2}^{2}\right| \leq \begin{cases}1-\mu & \text { if } \mu \leq \frac{2}{3} \\ \frac{1}{3} & \text { if } \frac{2}{3} \leq \mu \leq \frac{4}{3} \\ \mu-1 & \text { if } \mu \geq \frac{4}{3}\end{cases}
$$

This result was proved by Keogh and Merkes [12].

\section{Main Results}

Theorem 3. Let $f(z) \in C^{*}(A, B)$. Then,

$$
\left|a_{3}-\mu a_{2}^{2}\right| \leq \begin{cases}{[3+(A-B)(2-B)]-[2+(A-B)]^{2} \mu} & \text { if } \mu \leq \frac{-B}{2+(A-B)}(B<0, A+B<0) \\ {[3+(A-B)-4 \mu]+\frac{(A-B)(1-2 \mu)^{2}}{(1+B)+\mu(A-B)}} & \text { or } \mu \leq \frac{-B}{(A-B)}(B>0), \\ & \text { if } \frac{-B}{2+(A-B)} \leq \mu \leq \frac{1}{2} \\ 1+(A-B)+\frac{(A-B)(2 \mu-1)^{2}}{(1-B)-\mu(A-B)} & \text { if } \frac{1}{2+(A-B)} \leq \mu \leq \frac{-B}{(A-B)}(B<0), \\ (A-B)(4+A-B) \mu-[(A-B)(2-B)-1] & \text { if } \frac{2-B}{2+(A-B)} \leq \mu \leq \frac{1-B}{A-B}, \\ {[2+(A-B)]^{2} \mu-[3+(A-B)(2-B)]} & \text { if } \mu \geq \frac{1-B}{A-B} .\end{cases}
$$


These results are sharp.

Proof. By definition of $C^{*}(A, B)$,

$$
f(z)=g(z) \frac{1+A w(z)}{1+B w(z)}, \quad g \in S^{*}, w(z) \in \Omega
$$

Identifying terms in the above expansion,

$$
\begin{gathered}
a_{2}=b_{2}+(A-B) d_{1}, \\
a_{3}=b_{3}+(A-B) b_{2} d_{1}+(A-B)\left(d_{2}-B d_{1}^{2}\right) .
\end{gathered}
$$

From (15) and by using (2), it is easily established that

$$
\begin{aligned}
\left|a_{3}-\mu a_{2}^{2}\right| \leq & (A-B)+\left|b_{3}-\mu b_{2}^{2}\right|+(A-B)|1-2 \mu| x y \\
& +(A-B)(|B+\mu(A-B)|-1) x^{2},
\end{aligned}
$$

where $x=\left|d_{1}\right| \leq 1$ and $y=\left|b_{2}\right| \leq 2$. $B)$.

Two cases arise: (a) $\mu<-B /(A-B)$ and (b) $\mu \geq-B /(A-$

Consider the case $\mu<-B /(A-B)$.

Case $I$. Suppose that $\mu \leq 1 / 2$ so that $A+B \leq 0$.

By Lemma 1, (16) can be written as

$$
\begin{aligned}
&\left|a_{3}-\mu a_{2}^{2}\right| \leq {[3+(A-B)]-4 \mu+2(A-B) } \\
& \times(1-2 \mu) x-(A-B)[(1+B)+\mu(A-B)] x^{2} \\
&= H_{1}(x), \quad \text { say, } \\
& H_{1}^{\prime}(x)= 2(A-B)(1-2 \mu)-2(A-B) \\
& \times[(1+B)+\mu(A-B)] x, \\
& H_{1}^{\prime \prime}(x)=-2(A-B)[(1+B)+\mu(A-B)],
\end{aligned}
$$

$H_{1}^{\prime}(x)=0$ implies that $x=(1-2 \mu) /((1+B)+\mu(A-B))=x_{1}$. Subcase I(i). For $\mu<0, H_{1}^{\prime}(x)>0$ provided $\mu \leq-(1+B) /(A-$ $B)<-B /(A-B)$.

Where $H_{1}(x)$ is an increasing function in $[0,1]$ and $\max H_{1}(x)=H_{1}(1)$.

Subcase I(ii). Suppose $\mu \geq 0$ and $-(1+B) /(A-B) \leq \mu \leq$ $-B /(2+(A-B))$.

If $\mu<-B /(2+(A-B))$, then $H_{1}^{\prime}(x)>0$ and $\max H_{1}(x)=$ $H_{1}(1)$.

Combining the above two subcases, we obtain the first result of (13). For $x_{1}>1, H_{1}^{\prime \prime}(x)<0$ which holds for $\mu<-(1+B) /(A-B)$.

For $-(1+B) /(A-B) \leq \mu \leq-B /(2+(A-B))(B<0)$ or $-B /(2+(A-B)) \leq \mu \leq 1 / 2$ (for all $A$ and $B), \max H_{1}(x)=$ $H_{1}\left(x_{1}\right)$ from which the second result of (13) follows.

We now consider the case $\mu \geq-B /(A-B)$.
Case II. Suppose that $1 / 2 \leq \mu \leq 1$. Then by Lemma 1, (16) takes the following form:

$$
\begin{aligned}
\left|a_{3}-\mu a_{2}^{2}\right| \leq & 1+(A-B)+2(A-B)(2 \mu-1) x \\
& -(A-B)[(1-B)-\mu(A-B)] x^{2} \\
= & H_{2}(x),
\end{aligned}
$$

Subcase $I I(i)$. For $1 / 2 \leq \mu \leq(2-B) /(2+(A-B))$,

$$
\begin{aligned}
H_{2}^{\prime}(x)= & 2(A-B)(2 \mu-1) \\
& -2(A-B)[(1-B)-\mu(A-B)] x, \\
H_{2}^{\prime \prime}(x)= & -2(A-B)[(1-B)-\mu(A-B)],
\end{aligned}
$$

where $H_{2}^{\prime}(x)=0$ implies that $x=(2 \mu-1) /((1-B)-\mu(A-B))=$ $x_{2}$.

For $\mu \leq(2-B) /(2+(A-B))$, we have $x_{2}<1$ and $H_{2}^{\prime}(x)>0$. So $\max H_{2}(x)=H_{2}\left(x_{2}\right)$, which gives the third result of (13).

Subcase II(ii). For $(2-B) /(2+(A-B)) \leq \mu \leq(1-B) /(A-B)$. For $x_{2} \geq 1,(2-B) /(2+(A-B)) \leq \mu \leq(1-B) /(A-B), H_{2}^{\prime}(x)>$ 0 , and $\max H_{2}(x)=H_{2}(1)$, which leads us to the fourth result of (13).

Case III. For $\mu \geq(1-B) /(A-B) \geq 1$. By Lemma 1, (16) can be put in the following form:

$$
\begin{gathered}
\left|a_{3}-\mu a_{2}^{2}\right| \leq(A-B)+(4 \mu-3)+2(A-B)(2 \mu-1) x \\
-(A-B)[(1-B)-\mu(A-B)] x^{2} \\
=H_{3}(x), \quad \text { say, } \\
H_{3}^{\prime}(x)=2(A-B)(2 \mu-1)-2(A-B) \\
\quad \times[(1-B)-\mu(A-B)] x, \\
H_{3}^{\prime \prime}(x)=-2(A-B)[(1-B)-\mu(A-B)],
\end{gathered}
$$

where $H_{3}^{\prime}(x)=0$ shows that $x=(2 \mu-1) /((1-B)-\mu(A-B))=$ $x_{3}$.

If $x_{3}>1$, then $(2-B) /(2+(A-B))<\mu$, which is true. $H_{3}^{\prime}(x)>0$ for $\mu \geq(1-B) /(A-B) \geq 1$. So $\max H_{3}(x)=$ $\mathrm{H}_{3}(1)$, which takes us straight to the fifth result of (13).

The equality holds in the first and fifth results of (13) for the function $f_{1}(z)$ defined by

$$
f_{1}(z)=\frac{z}{(1-z)^{2}}\left(\frac{1+A z}{1+B z}\right) .
$$

The equality holds in the second result of (13) for the function $f_{2}(z)$ defined by

$$
f_{2}(z)=\frac{z}{(1-z)^{2}}\left(\frac{1+\left(A+x_{1}\right) z+A z^{2}}{1+\left(B+x_{1}\right) z+B z^{2}}\right),
$$

where $x_{1}=(1-2 \mu) /((1+B)+\mu(A-B))$. 
The equality holds in the third result of (13) for the function $f_{3}(z)$ defined by

$$
f_{3}(z)=\frac{z}{(1-z)^{2}}\left(\frac{1+\left(A+x_{2}\right) z+A z^{2}}{1+\left(B+x_{2}\right) z+B z^{2}}\right),
$$

where $x_{2}=(2 \mu-1) /((1-B)-\mu(A-B))$.

The equality holds in the fourth result of (13) for the function $f_{4}(z)$ defined by

$$
f_{4}(z)=\left(\int_{0}^{z}\left[1+\left(\frac{M^{2}-2 L}{M}\right) z\right]^{M^{2} /\left(M^{2}-2 L\right)} d z\right),
$$

where $L=3[(A-B)(2-B)-1]$ and $M=$ $2[(A-B)(4+A-B)]^{1 / 2}$ for $\left|\left(M^{2}-2 L\right) / M\right| \leq 1$.

Proof of Theorem 3 is completed.
For $A=1$ and $B=-1$, Theorem 3 gives the following result.

Corollary 4. If $f(z) \in C^{*}$, then,

$$
\left|a_{3}-\mu a_{2}^{2}\right| \leq \begin{cases}9-16 \mu & \text { if } \mu \leq \frac{1}{4}, \\ 1+\frac{1}{\mu} & \text { if } \frac{1}{4} \leq \mu \leq \frac{1}{2} \\ 3+\frac{(2 \mu-1)^{2}}{1-\mu} & \text { if } \frac{1}{2} \leq \mu \leq \frac{3}{4} \\ 12 \mu-5 & \text { if } \frac{3}{4} \leq \mu \leq 1, \\ 16 \mu-9 & \text { if } \mu \geq 1 .\end{cases}
$$

Theorem 5. Let $f(z) \in C_{1}^{*}(A, B)$. Then,

$$
\begin{aligned}
& \left|a_{3}-\mu a_{2}^{2}\right| \\
& \leq \begin{cases}{[1+(A-B)(1-B)]-[1+A-B]^{2} \mu} & \text { if } \mu \leq \frac{-(1+2 B)}{2(1+A-B)}(A+B>0), \\
{[1+(A-B)-\mu]+\frac{(A-B)(1-2 \mu)^{2}}{4[(1+B)+\mu(A-B)]}} & \text { if } \frac{-(1+2 B)}{2(1+A-B)} \leq \mu \leq \frac{-B}{A-B} \\
{[1+(A-B)-\mu]+\frac{(A-B)(2 \mu-1)^{2}}{4[(1-B)-\mu(A-B)]}} & \text { or } \frac{-(1+2 B)}{2(1+A-B)} \leq \mu \leq \frac{1}{2}, \\
\frac{1}{A-B} \leq \mu \leq \frac{2}{3}, \\
{[1+3(A-B)]+\frac{(A-B)(2 \mu-1)^{2}}{4[(1-B)-\mu(A-B)]}} & \text { if } \frac{2}{3} \leq \mu \leq \frac{3-2 B}{2(1+A-B)}, \\
\frac{1}{3}-(A-B)(1-B)+(A-B)(2+A-B) \mu & \text { if } \frac{3-2 B}{2(1+A-B)} \leq \mu \leq \frac{4}{3}, \\
{[1+A-B]^{2} \mu-[1+(A-B)(1-B)]} & \text { if } \mu \geq \frac{4}{3} .\end{cases}
\end{aligned}
$$

The results are sharp.

Proof. Proceeding as in Theorem 3, we have

$$
\begin{aligned}
\left|a_{3}-\mu a_{2}^{2}\right| \leq & (A-B)+\left|c_{3}-\mu c_{2}^{2}\right|+(A-B)|1-2 \mu| x y \\
& +(A-B)(|B+\mu(A-B)|-1) x^{2},
\end{aligned}
$$

where $x=\left|d_{1}\right| \leq 1$ and $y=\left|c_{2}\right| \leq 1$.

Two cases arise: (a) $\mu<-B /(A-B)$, (b) $\mu \geq-B /(A-B)$.

Consider the case $\mu<-B /(A-B)$.

By Lemma 2, (27) can be written as

$$
\begin{aligned}
\left|a_{3}-\mu a_{2}^{2}\right| \leq & {[1+(A-B)]-\mu+(A-B)|1-2 \mu| x } \\
& -(A-B)[(1+B)+\mu(A-B)] x^{2} .
\end{aligned}
$$

Subcase I(i). For $\mu<0$,

$$
\begin{aligned}
\left|a_{3}-\mu a_{2}^{2}\right| \leq & {[1+(A-B)]-\mu } \\
& +(A-B)(1-2 \mu) x-(A-B) \\
& \times[(1+B)+\mu(A-B)] x^{2}=H_{1}(x), \\
H_{1}^{\prime}(x)= & (A-B)(1-2 \mu) \\
& -2(A-B)[(1+B)+\mu(A-B)] x, \\
H_{1}^{\prime \prime}(x)= & -2(A-B)[(1+B)+\mu(A-B)] .
\end{aligned}
$$

For $\mu<0, H_{1}^{\prime}(x)>0$ which shows that $H_{1}(x)$ is an increasing function in $[0,1]$ and $\max H_{1}(x)=H_{1}(1)$. 
Subcase I(ii). Suppose $\mu \geq 0$.

$H_{1}^{\prime}(x)=0$ implies that $x=(1-2 \mu) /(2[(1+B)+\mu(A-$ $B)])=x_{1}$.

For $x_{1} \geq 1,-(1+B) /(A-B) \leq \mu \leq-(1+2 B) /(2(1+(A-$ $B)))$. So $H_{1}^{\prime}(x)>0$ and $\max H_{1}(x)=H_{1}(1)$.

Combining the above two subcases, we obtain the first result of (26).

For $x_{1}>1, H_{1}^{\prime \prime}(x)<0$, which holds for $-(1+2 B) /(2(1+$ $(A-B))) \leq \mu \leq-B /(A-B)$.

So $\max H_{1}(x)=H_{1}\left(x_{1}\right)$, which leads us to the second result of (26).

We now consider the case $\mu \geq-B /(A-B)$.

Subcase $I$ (iii). For $1 / 2 \leq-B /(A-B) \leq \mu \leq(2 / 3)(B<0,2 A+$ $B>0)$, (27) takes the following form:

$$
\begin{aligned}
\left|a_{3}-\mu a_{2}^{2}\right| \leq 1+ & (A-B)-\mu+(A-B)(2 \mu-1) x-(A-B) \\
\times & {[(1-B)-\mu(A-B)] x^{2}=H_{2}(x), } \\
H_{2}^{\prime}(x)= & (A-B)(2 \mu-1) \\
& -2(A-B)[(1-B)-\mu(A-B)] x, \\
H_{2}^{\prime \prime}(x)= & -2(A-B)[(1-B)-\mu(A-B)],
\end{aligned}
$$

where $H_{2}^{\prime}(x)=0$ implies that $x=(2 \mu-1) /(2[(1-B)-\mu(A-$ $B)])=x_{2}$.

It is easy to verify that $H_{2}^{\prime}(x)<0$. So $\max H_{2}(x)=H_{2}\left(x_{2}\right)$, which gives the third result of (26).

Case II. For $2 / 3 \leq \mu \leq 4 / 3$.

Subcase $I I(i)$. For $2 / 3 \leq \mu \leq(3-2 B) /(2(1+A-B))$, (27) reduces to

$$
\begin{aligned}
\left|a_{3}-\mu a_{2}^{2}\right| \leq \frac{1}{3}+ & (A-B)+(A-B)(2 \mu-1) x \\
& -(A-B)[(1-B)-\mu(A-B)] x^{2}=H_{3}(x), \\
H_{3}^{\prime}(x)= & (A-B)(2 \mu-1) \\
& -2(A-B)[(1-B)-\mu(A-B)] x \\
H_{3}^{\prime \prime}(x)= & -2(A-B)[(1-B)-\mu(A-B)]
\end{aligned}
$$

where $H_{3}^{\prime}(x)=0$ implies that $x=(2 \mu-1) /(2[(1-B)-\mu(A-$ $B)])=x_{3}$.

For $x_{3}<1,(2 / 3) \leq \mu \leq(3-2 B) /(2(1+A-B)), H_{3}^{\prime \prime}(x)<0$, and $\max H_{3}(x)=H_{3}\left(x_{3}\right)$, which leads us to the fourth result of (26).
Subcase II(ii). For $(3-2 B) /(2(1+A-B)) \leq \mu \leq 1$, (27) reduces to the following form:

$$
\begin{aligned}
\left|a_{3}-\mu a_{2}^{2}\right| \leq & \frac{1}{3}+(A-B)+(A-B)(2 \mu-1) x \\
& -(A-B)[(1-B)-\mu(A-B)] x^{2}=H_{4}(x), \\
H_{4}^{\prime}(x)= & (A-B)(2 \mu-1) \\
& -2(A-B)[(1-B)-\mu(A-B)] x
\end{aligned}
$$

$H_{4}^{\prime}(x)=0$ implies that $x=(2 \mu-1) /(2[(1-B)-\mu(A-B)])$. $\max H_{4}(x)=H_{4}(1)$.

Combining the above two subcases, $\max H_{4}(x)=H_{4}(1)$, which take us straight to the fifth result of (26).

Case III. For $\mu \geq 4 / 3$. By Lemma 1, (27) can be put in the form

$$
\begin{aligned}
\left|a_{3}-\mu a_{2}^{2}\right| \leq & (A-B)-1+\mu+(A-B)(2 \mu-1) x \\
& -(A-B)[(1-B)-\mu(A-B)] x^{2} \\
= & H_{5}(x), \\
H_{5}^{\prime}(x)= & (A-B)(2 \mu-1) \\
& -2(A-B)[(1-B)-\mu(A-B)] x,
\end{aligned}
$$

where $H_{5}^{\prime}(x)=0$ shows that $x=(2 \mu-1) /(2[(1-B)-\mu(A-$ $B)])=x_{5}$.

$x_{5}>1$ and $\max H_{5}(x)=H_{5}(1)$, which yield the sixth result of (26).

The equality holds in the first and sixth results of (26) for the function $f_{1}(z)$ defined by

$$
f_{1}(z)=\frac{z}{1-z}\left(\frac{1+A z}{1+B z}\right) .
$$

The equality holds in the second result of (26) for the function $f_{2}(z)$ defined by

$$
f_{2}(z)=\frac{z}{1-z}\left(\frac{1+\left(A+x_{1}\right) z+A z^{2}}{1+\left(B+x_{1}\right) z+B z^{2}}\right),
$$

where $x_{1}=(1-2 \mu) /(2[(1+B)+\mu(A-B)])$.

The equality holds in the third and fourth results of (26) for the function $f_{3}(z)$ defined by

$$
f_{3}(z)=\frac{z}{1-z}\left(\frac{1+\left(A+x_{2}\right) z+A z^{2}}{1+\left(B+x_{2}\right) z+B z^{2}}\right),
$$

where $x_{2}=(2 \mu-1) /(2[(1-B)-\mu(A-B)])$.

The equality holds in the fifth result of (26) for the function $f_{4}(z)$ defined by

$$
f_{4}(z)=\left(\int_{0}^{z}\left[1+\left(\frac{M^{2}-2 L}{M}\right) z\right]^{M^{2} /\left(M^{2}-2 L\right)} d z\right)
$$

where $L=1-3(A-B)(1-B)$ and $M=$ $2[(A-B)(2+A-B)]^{1 / 2}$ for $\left|\left(M^{2}-2 L\right) / M\right| \leq 1$.

Proof of Theorem 5 is completed. 
For $A=1, B=-1$, Theorem 5 gives the following result.

Corollary 6. If $f(z) \in C_{1}^{*}$, then

$$
\left|a_{3}-\mu a_{2}^{2}\right| \leq \begin{cases}5-9 \mu & \text { if } \mu \leq \frac{1}{6}, \\ 2+\frac{1}{4 \mu} & \text { if } \frac{1}{6} \leq \mu \leq \frac{1}{2}, \\ 3-\mu+\frac{(2 \mu-1)^{2}}{4(1-\mu)} & \text { if } \frac{1}{2} \leq \mu \leq \frac{2}{3}, \\ \frac{7+\frac{(2 \mu-1)^{2}}{4(1-\mu)}}{3+\frac{11}{3}} & \text { if } \frac{2}{3} \leq \mu \leq \frac{5}{6}, \\ 8 \mu-\frac{5}{6} \leq \mu \leq \frac{4}{3}, & \text { if } \frac{5}{6} .\end{cases}
$$

The results of Corollaries 4 and 6 were proved by the authors of [13].

\section{References}

[1] Z. Nehari, Conformal Mapping, McGraw-Hill Book, New York, NY, USA, 1952.

[2] L. Bieberbach, Uber Koeffizienten Derjenigen Potenzreihen, Welche Eine Schlichte Des Einheitskreises Vermitteln, vol. 38, Sitzungsberichte der Preußischen Akademie der Wissenschaften zu Berlin, 1916.

[3] L. de Branges, "A proof of the Bieberbach conjecture," Acta Mathematica, vol. 154, no. 1-2, pp. 137-152, 1985.

[4] K. Löwner, "Untersuchungen über schlichte konforme Abbildungen des Einheitskreises. I," Mathematische Annalen, vol. 89, no. 1-2, pp. 103-121, 1923.

[5] M. Fekete and G. Szegö, "Eine Bemer Kung uber ungerade schlichte Functionen," Journal of the London Mathematical Society, vol. 8, pp. 85-89, 1933.

[6] P. N. Chichra, "New subclasses of the class of close-to-convex functions," Proceedings of the American Mathematical Society, vol. 62, no. 1, pp. 37-43, 1977.

[7] K. O. Babalola, "The fifth and sixth coefficients of $\alpha$-close-toconvex functions," Kragujevac Journal of Mathematics, vol. 32, pp. 5-12, 2009.

[8] M. O. Reade, "On close-to-close univalent functions," The Michigan Mathematical Journal, vol. 3, pp. 59-62, 1955.

[9] B. S. Mehrok, G. Singh, and D. Gupta, "A subclass of analytic functions," Global Journal of Mathematical Sciences, vol. 2, no. 1, pp. 91-97, 2010.

[10] B. S. Mehrok, G. Singh, and D. Gupta, "On a subclass of analytic functions," Antarctica Journal of Mathematics, vol. 7, no. 4, pp. 447-453, 2010.

[11] J. A. Hummel, "The coefficient regions of starlike functions," Pacific Journal of Mathematics, vol. 7, pp. 1381-1389, 1957.

[12] F. R. Keogh and E. P. Merkes, "A coefficient inequality for certain classes of analytic functions," Proceedings of the American Mathematical Society, vol. 20, pp. 8-12, 1969.

[13] B. S. Mehrok and G. Singh, "Fekete-Szegö inequality for two subclasses of analyticfunctions," In press. 


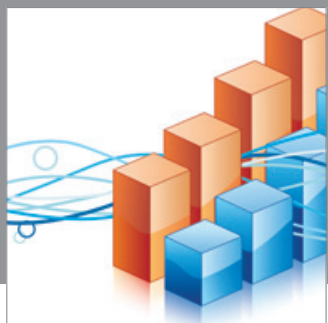

Advances in

Operations Research

mansans

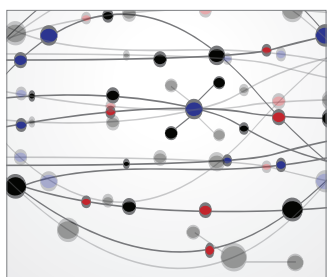

The Scientific World Journal
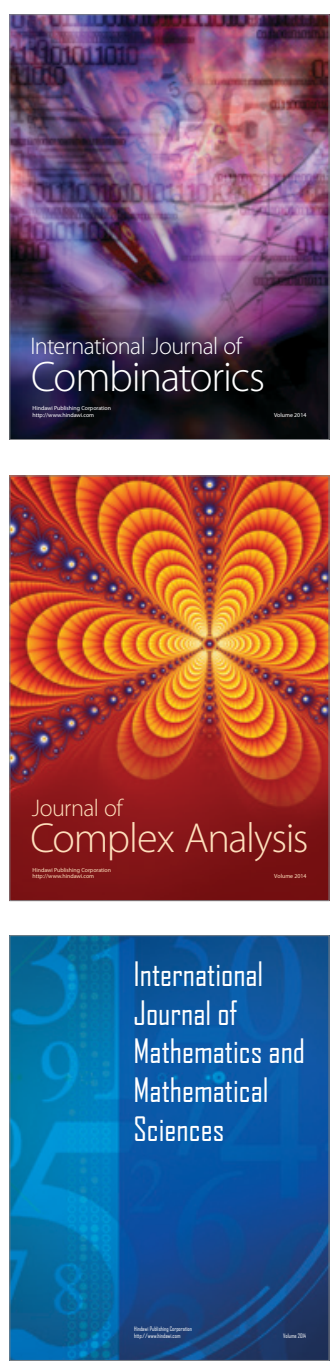
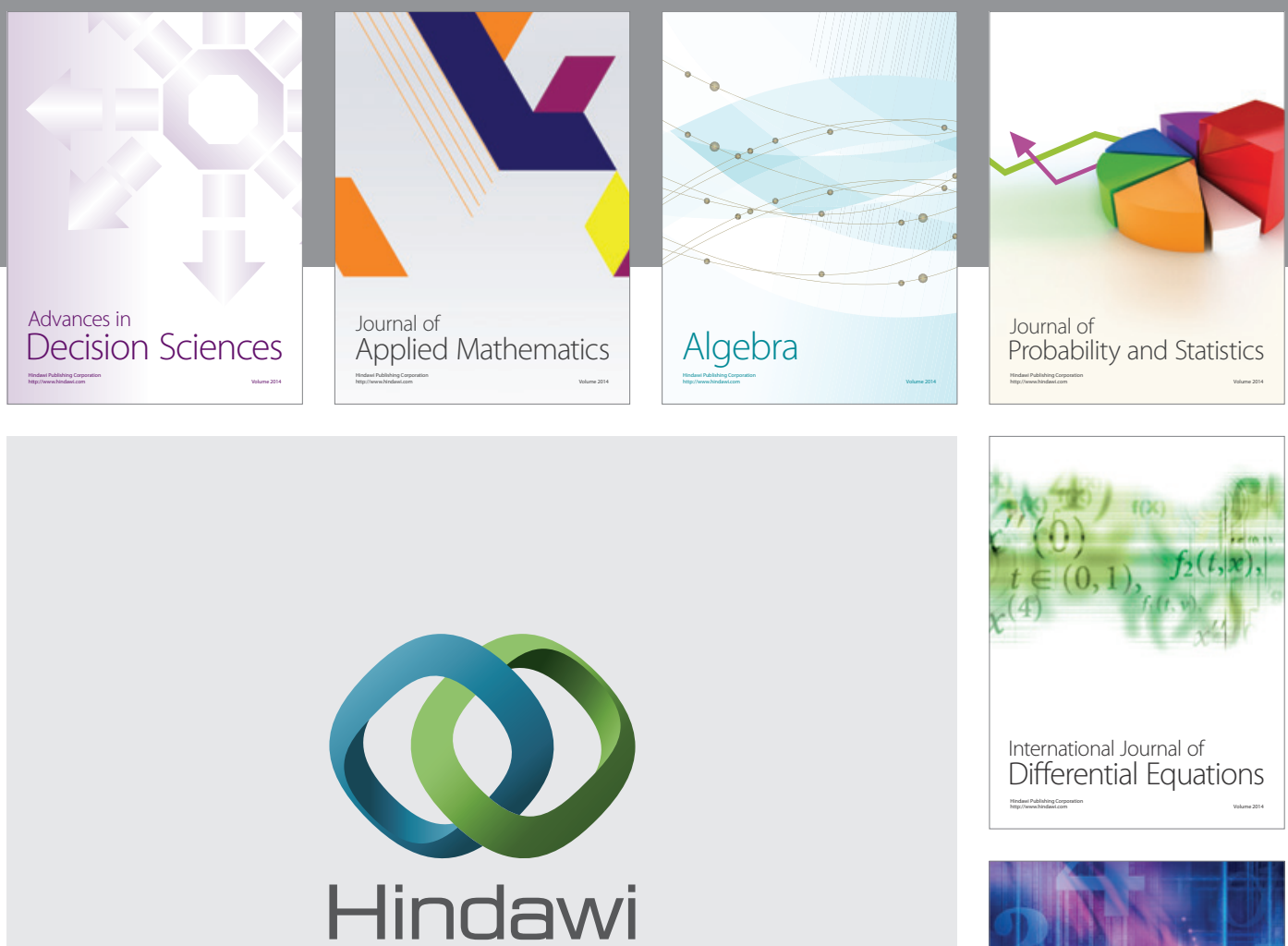

Submit your manuscripts at http://www.hindawi.com
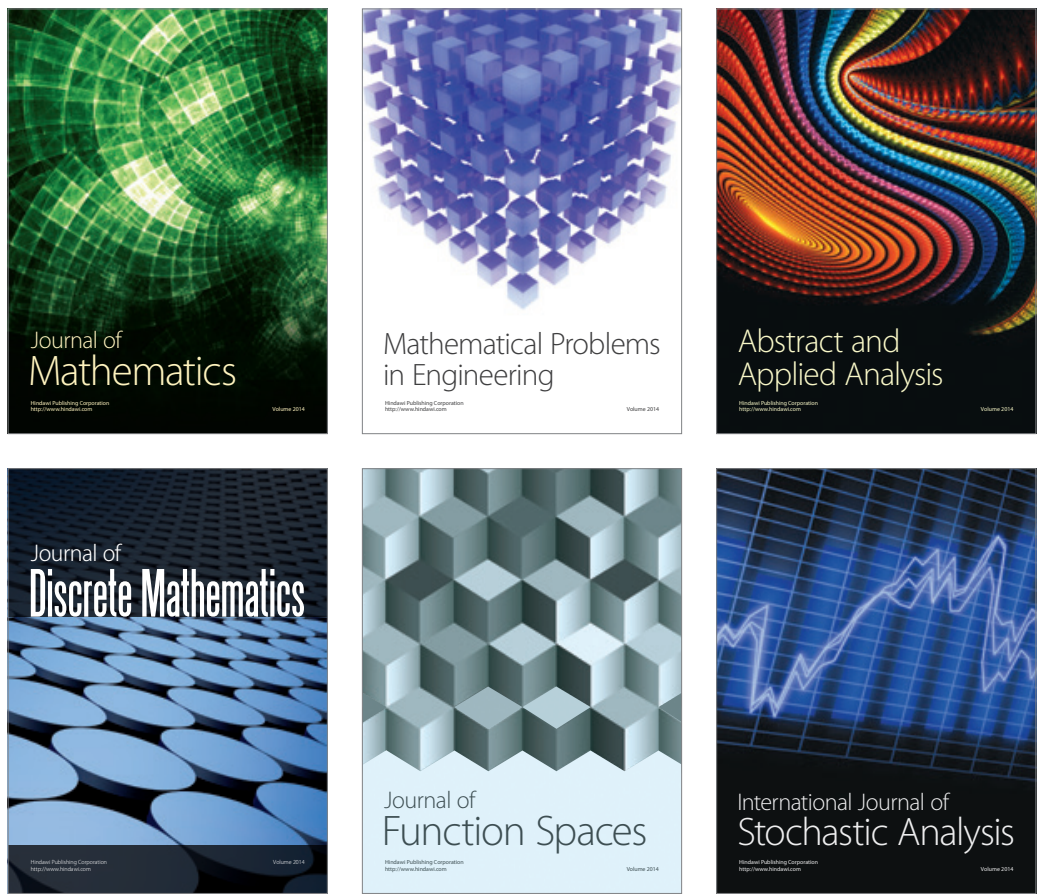

Journal of

Function Spaces

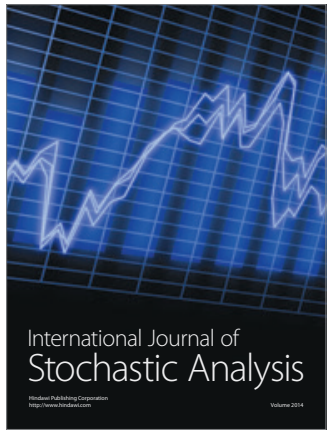

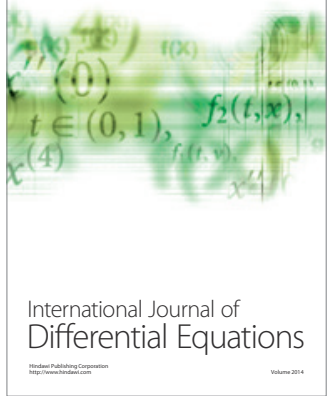
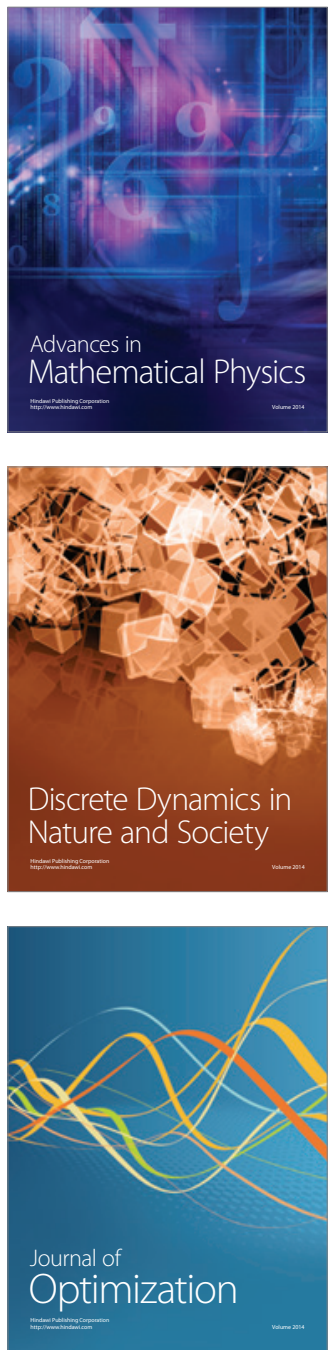